\title{
AVALIAÇÃO DE MÉTODOS DE ESTIMATIVA DA EVAPOTRANSPIRAÇÃO HORÁRIA PARA ALFACE CULTIVADA EM SISTEMA HIDROPÔNICO EM AMBIENTE PROTEGIDO
}

\author{
CARLOS R. W. MOURA ${ }^{1}$, SÉRGIO ZOLNIER ${ }^{2}$, ARISTIDES RIBEIRO ${ }^{3}$, \\ RUBENS A. DE OLIVEIRA ${ }^{4}$
}

RESUMO: O objetivo deste trabalho foi avaliar a precisão e a exatidão de métodos de estimativa de valores horários de evapotranspiração $\left(E_{c}\right)$ para alface cultivada em sistema hidropônico sob ambiente protegido. Para a determinação dos parâmetros pertinentes aos diferentes métodos de estimativa e sua posterior validação, foram utilizados dois conjuntos de dados experimentais obtidos a partir de medições realizadas nas cultivares Grand Rapids, Regina e Great Lakes. As estimativas de evapotranspiração foram realizadas com os seguintes métodos: Penman-Monteith parametrizado pela FAO, em 1998 (PMF); Penman-Monteith modificado para condições aerodinâmicas características de ambiente protegido (PMAP); Penman-Monteith modificado para ambiente protegido simplificado (PMAPS); Priestley-Taylor (PT); Radiação solar (RS) e Radiação solar simplificado (RSS). Os resultados demonstraram que os métodos PMAP e PMAPS possibilitaram estimativas mais adequadas da $\mathrm{ET}_{\mathrm{c}}$, tanto no período da manhã como no da tarde.

PALAVRAS-CHAVE: cultivo protegido, horticultura, técnica de circulação laminar de nutrientes.

\section{EVALUATION OF METHODS OF ESTIMATION OF HOURLY EVAPOTRANSPIRATION FOR HYDROPONIC LETTUCE UNDER PROTECTED ENVIRONMENT}

\begin{abstract}
The objective of this work was to evaluate the precision and accuracy of different methods for estimating hourly values of evapotranspiration $\left(\mathrm{ET}_{\mathrm{c}}\right)$ for hydroponic lettuce under protected environment conditions. To determine the necessary parameters for different estimating methods, and their subsequent validation, two sets of experimental data were obtained from measurements carried out with cultivars Grand Rapids, Regina and Great Lakes. Evapotranspiration estimates were conducted with the following methods: Penman-Monteith parameterized by FAO in 1998 (PMF); Penman-Monteith modified for aerodynamic characteristics in protected environment (PMAP); Simplified Penman-Monteith (PMAPS); Priestley-Taylor (PT); Solar radiation (RS) and Simplified solar radiation (RSS). The results demonstrated that the PMAP and PMAPS methods allowed better estimates of the hourly values of ETc, both in the morning and in the afternoon period.
\end{abstract}

KEYWORDS: protected cultivation, horticulture, nutrient film technique.

\footnotetext{
${ }^{1}$ Meteorologista, Mestre, Centro de Previsão de Tempo e Estudos Climáticos - CPTEC, INPE, Av. dos Astronautas, 1758, São José dos Campos - SP, carloswmoura@ hotmail.com.

${ }^{2}$ Eng $^{\text {o }}$ Agrícola, Ph.D., Departamento de Engenharia Agrícola, Universidade Federal de Viçosa, Viçosa - MG, zolnier@ufv.br.

${ }^{3}$ Eng $^{\mathrm{O}}$ Agrônomo, Doutor, Departamento de Engenharia Agrícola, Universidade Federal de Viçosa, Viçosa - MG, ribeiro@ ufv.br.

${ }^{4}$ Eng ${ }^{\circ}$ Agrícola, Doutor, Departamento de Engenharia Agrícola, Universidade Federal de Viçosa, Viçosa - MG, rubens@ufv.br.

Recebido pelo Conselho Editorial em: 31-1-2008

Aprovado pelo Conselho Editorial em: 20-5-2010
} 


\section{INTRODUÇÃO}

A produção e o consumo de alface cultivada em sistemas hidropônicos do tipo NFT ("nutrient film technique") aumentaram consideravelmente nos últimos anos no Brasil (GUALBERTO et al., 2009). Os principais fatores que proporcionam o aumento de produção estão associados à redução do ciclo da cultura, menor incidência de pragas e doenças, maior eficiência do uso de água e de fertilizantes e possibilidade de aproveitamento de pequenas áreas próximas aos centros consumidores (FELTRIN et al., 2005; SILVA et al., 2005; LUZ et al., 2006; MAGGI et al., 2006). As principais desvantagens desta técnica de cultivo estão relacionadas ao elevado custo inicial para sua implantação, à falta de automação do processo de preparo da solução nutritiva e ao empirismo associado à frequência de aplicação da solução nutritiva (LUZ et al., 2006; ZOLNIER et al., 2004).

A aplicação da solução nutritiva em sistemas hidropônicos é um aspecto importante a ser considerado, pois, se mal manejada, além do desiquilíbrio nutricional, pode limitar a disponibilidade de oxigênio na superfície das raízes, favorecer a ocorrência de doenças no sistema radicular e causar estresse hídrico ao longo do ciclo da cultura (JONES, 1997). Basicamente, duas técnicas de controle são adotadas para a aplicação da solução nutritiva, sendo uma por meio de temporizadores e outra baseada na estimativa de evapotranspiração. Em decorrrência da ausência de substrato no sistema NFT de cultivo, as estimativas de evapotranspiração precisam ser realizadas em curtos períodos de tempo, necessitando, assim, de monitoramento contínuo das variáveis meteorológicas no ambiente de cultivo.

De acordo com ZOLNIER et al. (2004), a principal desvantagem do uso de temporizadores está relacionada à necessidade de se adotarem valores fixos para o controle da duração e dos intervalos de aplicação da solução nutritiva. No caso da alface, usualmente, a solução é recirculada durante um período 10 a 15 min, mantendo-se intervalos de 15 a 20 min entre eventos de aplicação. Em decorrência disso, essa técnica de controle não é capaz de realizar ajustes da frequência de aplicação da solução nutritiva, com as alterações das condições meteorológicas ao longo do dia e aumento do consumo de água, devido à expansão foliar, ao longo do período de crescimento.

Normalmente, a estimativa da evapotranspiração em sistemas hidropônicos é realizada por meio de modelos baseados no balanço de energia, destacando-se o de Penman-Monteith (MONTEITH, 1965). Contudo, uma das limitações deste método refere-se à estimativa de parâmetros específicos da cultura, como a resistência de superfície ao processo de difusão de vapor de água e a resistência aerodinâmica ao processo de difusão de calor sensível (LYRA et al., 2003). Por outro lado, para estimativas de evapotranspiração em escala de minutos, o modelo de Penman-Monteith requer medições da radiação solar, temperatura, umidade relativa e velocidade do ar, por meio de sensores eletrônicos e sistemas de aquisição de dados, o que dificulta a automação do sistema de cultivo em pequenas propriedades devido ao custo do equipamento.

Neste trabalho, teve-se por objetivo avaliar métodos alternativos para estimativa da evapotranspiração horária em sistema hidropônico do tipo NFT, a partir da determinação de índices estatísticos de precisão e exatidão, visando à redução do número de variáveis meteorológicas monitoradas eletronicamente para implementação de sistemas de baixo custo.

\section{MATERIAL E MÉTODOS}

\section{Métodos de estimativa da evapotranspiração de referência ou potencial}

\section{a) Penman-Monteith-FAO (PMF)}

Esse método é utilizado, frequentemente, para estimativa da evapotranspiração de uma cultura hipotética em condições de campo. Posteriormente, o valor de referência estimado é multiplicado pelo coeficiente de cultura $\left(\mathrm{K}_{\mathrm{c}}\right)$ para se obter a evapotranspiração ao longo do período de crescimento. Quando aplicado em sistemas hidropônicos, o fluxo de calor sensível corresponde ao aquecimento da solução nutritiva (ZOLNIER et al., 2004). Neste caso, tem-se: 


$$
\mathrm{LE}_{\mathrm{o}}=\frac{\Delta\left(\mathrm{R}_{\mathrm{n}}-\mathrm{F}\right)+\rho_{\mathrm{ar}} \mathrm{c}_{\mathrm{par}} \mathrm{DPV} \mathrm{ar}_{\mathrm{ar}} / \mathrm{r}_{\mathrm{a}}}{\Delta+\gamma\left(1+\mathrm{r}_{\mathrm{s}} / \mathrm{r}_{\mathrm{a}}\right)}
$$

em que,

$\mathrm{LE}_{\mathrm{o}}$ - fluxo de calor latente de referência à superfície da cultura hipotética, $\mathrm{W} \mathrm{m}^{-2}$;

$\mathrm{R}_{\mathrm{n}}$ - saldo de radiação à superfície, $\mathrm{W} \mathrm{m} \mathrm{m}^{-2}$;

F - fluxo de calor sensível na solução nutritiva, normalizado para a área da bancada de cultivo, $\mathrm{W} \mathrm{m}^{-2}$

$\Delta$ - derivada da curva de pressão de saturação do vapor de água com respeito à temperatura, $\mathrm{Pa}^{\circ} \mathrm{C}^{-1}$;

$\rho_{\text {ar }}$ - densidade absoluta do ar, $\mathrm{kg} \mathrm{m}^{-3}$;

$\mathrm{c}_{\mathrm{par}}$ - calor específico do ar à pressão constante, $\mathrm{J} \mathrm{kg}^{-1}{ }^{\circ} \mathrm{C}^{-1}$;

$\mathrm{DPV}_{\mathrm{ar}}$ - déficit da pressão de saturação do vapor de água no ar, $\mathrm{Pa}$;

$\gamma$ - coeficiente psicrométrico, $\mathrm{Pa}^{\circ} \mathrm{C}^{-1}$;

$\mathrm{r}_{\mathrm{a}}$ - resistência ao fluxo de calor sensível, $\mathrm{s} \mathrm{m}^{-1}$, e

$\mathrm{r}_{\mathrm{s}}$ - resistência de superfície da cultura de referência, $\mathrm{s} \mathrm{m}^{-1}$.

No cálculo dos parâmetros necessários para aplicação deste método, foram utilizados os procedimentos propostos por ALLEN et al. (1998). Com base nessa metodologia, o termo $r_{a}$ é estimado pela relação 208/ $\mathrm{U}_{2}$, sendo $\mathrm{U}_{2}$ a velocidade do ar a dois metros de altura, expressa em $\mathrm{ms}^{-1}$.

\section{b) Penman-Monteith Modificado para Ambientes Protegidos (PMAP)}

Nesse método, a resistência ao fluxo de calor sensível da equação de Penman-Monteith-FAO (Equação 1) foi substituída conforme descrito a seguir, a partir da utilização do fator de desacoplamento $(\Omega)$ proposto por McNAUGHTON \& JARVIS (1983):

$$
\Omega=\frac{1}{1+\frac{\gamma}{\Delta+\gamma}\left[\frac{\mathrm{r}_{\mathrm{s}}}{\mathrm{r}_{\mathrm{a}}}\right]}
$$

De acordo com esses autores, o coeficiente de desacoplamento médio para uma superfície de grama com crescimento rasteiro, cobrindo totalmente o solo, bem irrigada e com albedo de 0,23 será aproximadamente 0,80. Aplicando-se na Equação 2 os valores $\Omega=0,80$, proposto pelos autores, e $\mathrm{r}_{\mathrm{s}}=70 \mathrm{~s} \mathrm{~m}^{-1}$, proposto por ALLEN et al. (1998), a resistência aerodinâmica poderá ser estimada por:

$$
r_{\mathrm{a}}=\frac{280 \gamma}{\gamma+\Delta}
$$

A vantagem da eq.(3), em relação à proposta por ALLEN et al. (1998), é que a resistência aerodinâmica não tende para o infinito quando a velocidade do ar tende para zero ou valores extremamente baixos, como os observados em ambiente protegido. Com a utilização do fator de desacoplamento, é importante observar que a velocidade do ar não é mais necessária para a estimativa do termo $r_{a}$. Finalmente, deve-se ressaltar também que, com exceção do parâmetro $r_{a}$, a equação utilizada para estimativa da evapotranspiração pelo método PMAP é a mesma adotada pelo método PMF (eq.(1)).

\section{c) PMAP Simplificado (PMAPS)}

Devido à queda acentuada da temperatura do ar no período noturno e a condições microclimáticas específicas de ambientes protegidos, a umidade relativa do ar alcança valores próximos de $100 \%$ ao nascer do sol (FARIAS et al., 1992; BURIOL et al., 2000). Assim, considerando-se essa especificidade meteorológica, o $\mathrm{DPV}_{\mathrm{ar}}$ ao longo do período diurno pode ser estimado pela diferença entre a pressão de saturação de vapor de água $\left(\mathrm{e}_{\mathrm{s}}\right)$, determinada a partir da temperatura do ar média do período de estimativa da evapotranspiração, e a pressão de saturação ( $\left.\mathrm{e}_{\mathrm{s} \_ \text {min }}\right)$, calculada com a temperatura mínima do ar medida ao nascer do sol. Dessa forma, o método PMAP foi simplificado, conforme equação apresentada a seguir: 


$$
\mathrm{LE}_{\mathrm{o}}=\frac{\Delta\left(\mathrm{R}_{\mathrm{n}}-\mathrm{F}\right)+\rho_{\mathrm{ar}} \mathrm{c}_{\mathrm{par}}\left(\mathrm{e}_{\mathrm{s}}-\mathrm{e}_{\mathrm{s}_{-} \min }\right) / \mathrm{r}_{\mathrm{a}}}{\Delta+\gamma\left(1+\mathrm{r}_{\mathrm{s}} / \mathrm{r}_{\mathrm{a}}\right)}
$$

Deve-se observar que esse método não necessita de medições da velocidade do ar e umidade relativa no ambiente de cultivo, em decorrência do uso do fator de desacoplamento e da simplificação descrita anteriormente.

d) Priestley-Taylor (PT)

A equação de Priestley-Taylor pode ser interpretada como uma simplificação do método originalmente proposto por Penman, onde a evapotranspiração potencial ( $\left.\mathrm{LE}_{\mathrm{p}}\right)$, expressa também em W m ${ }^{-2}$, é estimada exclusivamente a partir da evapotranspiração de equilíbrio (McNAUGHTON $\&$ JARVIS (1983). Nesse caso, um fator de ajuste $\alpha$, universalmente conhecido como parâmetro de Priestley-Taylor, compensa os efeitos do termo aerodinâmico que são ignorados pelo método:

$$
\mathrm{LE}_{\mathrm{p}}=\alpha \frac{\Delta}{\Delta+\gamma}\left(\mathrm{R}_{\mathrm{n}}-\mathrm{F}\right)
$$

É importante notar que, para a utilização da eq.(5), são necessários dados da temperatura do ar, da solução nutritiva para estimativa da variável $F$ e medições ou estimativas do saldo de radiação. O parâmetro alfa é obtido a partir de uma calibração do método com um conjunto independente de dados para um sistema de cultivo específico.

\section{e) Radiação Solar (RS)}

Esse método requer apenas medições da radiação solar global incidente $\left(\mathrm{R}_{\mathrm{g}}\right)$, expressa em $\mathrm{W} \mathrm{m}^{-2}$, temperatura do ar no ambiente interno e calibração preliminar para a determinação do fator de correção (r), nesse caso, específico do ambiente protegido, sendo dado, segundo PEREIRA et al. (1997), por:

$$
\mathrm{LE}_{\mathrm{p}}=\mathrm{r} \frac{\Delta}{\Delta+\gamma} \mathrm{R}_{\mathrm{g}}
$$

\section{f) Radiação Solar Simplificado (RSS)}

Esse método é bastante similar ao da radiação solar, no entanto a variável $\mathrm{R}_{\mathrm{g}}$ é estimada pela equação proposta por HARGREAVES \& SAMANI (1982). Para possibilitar a estimativa de $R_{\mathrm{g}}$ ao longo do período diurno, em escala horária, a temperatura máxima diária foi substituída pela temperatura média do ar no período de estimativa considerado.

$$
\mathrm{LE}_{\mathrm{p}}=\mathrm{r} \frac{\Delta}{\Delta+\gamma} \mathrm{k}_{\mathrm{r}} \sqrt{\mathrm{t}_{\mathrm{m}}-\mathrm{t}_{\min }} \mathrm{R}_{\mathrm{a}}
$$

em que,

$\mathrm{k}_{\mathrm{r}}$ - coeficiente de ajuste, ${ }^{\circ} \mathrm{C}^{-0,5}$;

$\mathrm{t}_{\mathrm{m}}$ - temperatura do ar média para o período considerado, ${ }^{\circ} \mathrm{C}$;

$\mathrm{t}_{\min }$ - temperatura do ar mínima diária, ${ }^{\circ} \mathrm{C}$, e

$\mathrm{R}_{\mathrm{a}}$ - radiação solar instantânea no topo da atmosfera, $\mathrm{W} \mathrm{m}^{-2}$.

\section{Avaliação dos métodos de estimativa da evapotranspiração}

Após a determinação dos parâmetros necessários para a implementação dos métodos descritos anteriormente, foi utilizado outro conjunto de valores medidos das variáveis meteorológicas para estimativa da evapotranspiração da alface $\left(\mathrm{ET}_{\mathrm{c}}\right)$ ao longo do ciclo de crescimento. A $\mathrm{ET}_{\mathrm{c}}$ foi obtida a partir da multiplicação do coeficiente de cultura $\mathrm{K}_{\mathrm{c}}$ pela evapotranspiração de referência $\left(\mathrm{LE}_{\mathrm{o}}\right)$ ou potencial $\left(\mathrm{LE}_{\mathrm{p}}\right)$. Em seguida, os valores estimados foram comparados com os valores medidos. Para teste de desempenho dos métodos de estimativa da evapotranspiração, foram determinados vários índices estatísticos de precisão (coeficiente de correlação, r) e de exatidão (índice de concordância, d). Adicionalmente, foram estimados os seguintes erros estatísticos: erro médio de estimativa (MBE) e a raiz quadrada do quadrado do erro médio de estimativa (RMSE). As equações utilizadas 
para estimativa de cada um dos índices estatísticos e erros associados podem ser encontradas em JACOVIDES \& KONTOYIANNIS (1995).

\section{Medições de evapotranspiração e das variáveis meteorológicas em ambiente protegido}

Para a avaliação dos métodos de estimativa da evapotranspiração descritos previamente, foram utilizadas medições do consumo de água e das variáveis ambientais obtidas em dois experimentos independentes. As medições de $\mathrm{ET}_{\mathrm{c}}$ foram realizadas nas cultivares de alface Grand Rapids, Regina e Great Lakes em sistema hidropônico do tipo NFT sob ambiente protegido. Os experimentos foram conduzidos na área experimental da Meteorologia Agrícola, pertencente ao Departamento de Engenharia Agrícola (DEA), no Câmpus da Universidade Federal de Viçosa (UFV), Viçosa-MG, de coordenadas geográficas: latitude $20^{\circ} 45^{\prime} \mathrm{S}$, longitude $42^{\circ} 51^{\prime} \mathrm{W}$ e altitude $690 \mathrm{~m}$.

A área da superfície vegetal das plantas foi determinada periodicamente, utilizando-se de um medidor de área foliar (Modelo LI-3100, LI-COR, Inc., Lincoln, NE). Em cada amostragem, foram colhidas dez plantas por cultivar. As plantas utilizadas para a determinação destrutiva da área foliar foram susbstituídas por plantas que estavam sendo cultivadas, paralelamente, na mesma casa de vegetação. A área foliar total das plantas amostradas foi dividida pela área ocupada na bancada de crescimento, permitindo, assim, a obtenção do índice de área foliar (IAF).

Os dados obtidos em um dos experimentos foram utilizados para a estimativa dos parâmetros dos modelos de evapotranspiração, incluindo $\alpha, \mathrm{r}$ e $\mathrm{k}_{\mathrm{r}}$, e o segundo conjunto foi usado para a validação dos modelos. Informações sobre as dimensões da casa de vegetação, sistema hidropônico, sistema de aquisição de dados e instrumentos utilizados para a medição das variáveis meteorológicas podem ser obtidas em LYRA (2002).

\section{RESULTADOS E DISCUSSÃO}

$\mathrm{Na}$ Tabela 1, encontram-se os valores do coeficiente de correlação (r), do índice de concordância (d), da raiz quadrada do quadrado do erro médio de estimativa (RMSE) e do erro médio de estimativa (MBE), referentes ao desempenho estatístico dos diferentes métodos utilizados para estimativa horária da evapotranspiração dos cultivares de alface Grand Rapids, Regina e Great Lakes em sistema hidropônico do tipo NFT sob ambiente protegido.

Os resultados foram organizados com o intuito de mostrar a variação de desempenho dos métodos ao longo do ciclo da cultura, que foi dividido em 5 etapas de medição a partir do transplantio. Cada etapa compreendeu um período de medição de três dias, os quais foram intercalados por períodos de dois dias quando a $\mathrm{ET}_{\mathrm{c}}$ da alface não foi obtida. A primeira etapa teve início no período compreendido entre o $3^{-}$e o $5^{\circ}$ dia após o tranplantio, quando o índice de área foliar (IAF) médio para os três cultivares alcançou 0,12 . Na segunda, terceira, quarta e quinta etapas, o IAF médio foi 0,26, 0,62, 1,64 e 3,14, respectivamente.

De forma geral, verifica-se que os coeficientes de precisão (r) e de exatidão (d) apresentaram valores em torno de 0,9 para os métodos PMAP e PMAPS, e que seus valores diminuíram à medida que as estimativas foram realizadas pelos métodos PMF ou pelos que utilizam somente dados de radiação solar e/ou temperatura do ar (PT, RS e RSS). Com relação aos coeficientes RMSE e MBE, observa-se que o erro de precisão de todos os métodos avaliados foi, no máximo, $10 \mathrm{~W} \mathrm{~m}^{-2}$ nas duas primeiras etapas de medição. Com o aumento da $\mathrm{ET}_{\mathrm{c}}$, resultante da expansão foliar, o erro de precisão aumentou nas etapas seguintes, ficando, respectivamente, entre 20 e $54 \mathrm{~W} \mathrm{~m}^{-2}$ para os métodos PMF, PMAP e PMAPS e entre 24 e $75 \mathrm{~W} \mathrm{~m}^{-2}$ para PT, RS e RSS. Com relação à exatidão, nota-se que, independentemente da cultivar, o MBE foi menor que $2 \mathrm{~W} \mathrm{~m}^{-2}$ nas duas primeiras etapas de medição quando foram utilizados os métodos PMAP e PMAPS. Para as etapas seguintes, o MBE foi positivo, com base nas estimativas obtidas com PMAP, PMAPS, PT e RS, indicando que esses métodos superestimaram a $\mathrm{ET}_{\mathrm{c}}$. Em contraste, o método PMF subestimou a ETc para a cultivar Great Lakes em todas as etapas de medição. 
TABELA 1. Desempenho estatístico dos métodos Penman-Monteith-FAO (PMF), Penman-Monteith Modificado para Ambientes Protegidos (PMAP), PMAP Simplificado (PMAPS), PriestleyTaylor (PT), Radiação Solar (RS) e RS Simplificado (RSS) para a estimativa da evapotranspiração das cultivares de alface Grand Rapids, Regina e Great Lakes em sistema hidropônico sob ambiente protegido. O período experimental compreendeu cinco etapas de medição, cada uma com duração de três dias, que foram conduzidas após o transplantio. Statistical Performance of the Methods Penman-Monteith-FAO (PMF), PenmanMonteith Modified for Greenhouse Conditions (PMAP), Simplified PMAP (PMAPS), Priestley-Taylor (PT), Solar Radiation (RS) and Simplified RS (RSS) for evapotranspiration estimates of the lettuce cultivars Grand Rapids, Regina and Great Lakes in hydroponic system under greenhouse conditions. The experimental period encompassed five measurement periods, each one of them consisting of three days, performed after transplanting.

\begin{tabular}{|c|c|c|c|c|c|c|c|c|c|c|c|c|c|}
\hline \multirow[b]{3}{*}{ Método } & \multirow[b]{3}{*}{ Etapas } & \multicolumn{12}{|c|}{ Cultivares } \\
\hline & & \multicolumn{4}{|c|}{ Grand Rapids } & \multicolumn{4}{|c|}{ Regina } & \multicolumn{4}{|c|}{ Great Lakes } \\
\hline & & $\mathrm{R}$ & d & $\begin{array}{l}\text { RMSE } \\
\left(\mathrm{W} \mathrm{m}^{-2}\right)\end{array}$ & $\begin{array}{c}\mathrm{MBE} \\
\left(\mathrm{W} \mathrm{m}^{-2}\right) \\
\end{array}$ & $\mathrm{r}$ & $\mathrm{D}$ & $\begin{array}{l}\text { RMSE } \\
\left(\mathrm{W} \mathrm{m}^{-2}\right)\end{array}$ & $\begin{array}{c}\mathrm{MBE} \\
\left(\mathrm{W} \mathrm{m}^{-2}\right) \\
\end{array}$ & $\mathrm{r}$ & d & $\begin{array}{l}\text { RMSE } \\
\left(\mathrm{W} \mathrm{m}^{-2}\right)\end{array}$ & $\begin{array}{c}\mathrm{MBE} \\
\left(\mathrm{W} \mathrm{m}^{-2}\right) \\
\end{array}$ \\
\hline \multirow{5}{*}{ PMF } & $1^{\mathrm{a}}$ & 0,82 & 0,82 & 8 & -5 & 0,79 & 0,79 & 7 & -5 & 0,70 & 0,70 & 10 & -7 \\
\hline & $2^{\underline{a}}$ & 0,88 & 0,88 & 9 & -5 & 0,87 & 0,91 & 6 & -3 & 0,87 & 0,86 & 9 & -6 \\
\hline & $3^{\mathrm{a}}$ & 0,91 & 0,95 & 21 & -4 & 0,92 & 0,97 & 20 & 10 & 0,90 & 0,94 & 19 & -2 \\
\hline & $4^{\mathrm{a}}$ & 0,86 & 0,92 & 36 & 11 & 0,86 & 0,94 & 34 & 9 & 0,87 & 0,93 & 31 & -6 \\
\hline & $5^{\mathrm{a}}$ & 0,88 & 0,93 & 43 & 11 & 0,87 & 0,98 & 40 & 7 & 0,89 & 0,94 & 36 & -1 \\
\hline \multirow{5}{*}{ PMAP } & $1^{\mathrm{a}}$ & 0,93 & 0,96 & 4 & 1 & 0,89 & 0,91 & 5 & -2 & 0,86 & 0,90 & 5 & -2 \\
\hline & $2^{\mathrm{a}}$ & 0,90 & 0,95 & 6 & 0 & 0,92 & 0,96 & 4 & 1 & 0,92 & 0,95 & 5 & -1 \\
\hline & $3^{\mathrm{a}}$ & 0,95 & 0,96 & 21 & 14 & 0,96 & 0,95 & 28 & 23 & 0,95 & 0,95 & 20 & 14 \\
\hline & $4^{\mathrm{a}}$ & 0,89 & 0,87 & 48 & 38 & 0,91 & 0,91 & 40 & 30 & 0,92 & 0,94 & 30 & 17 \\
\hline & $5^{\mathrm{a}}$ & 0,92 & 0,92 & 47 & 34 & 0,92 & 0,99 & 41 & 25 & 0,94 & 0,95 & 34 & 18 \\
\hline \multirow{5}{*}{ PMAPS } & $1^{\mathrm{a}}$ & 0,88 & 0,93 & 5 & -1 & 0,82 & 0,87 & 6 & -1 & 0,81 & 0,86 & 6 & -1 \\
\hline & $2^{\mathrm{a}}$ & 0,89 & 0,94 & 7 & 2 & 0,91 & 0,95 & 5 & 1 & 0,92 & 0,95 & 5 & 1 \\
\hline & $3^{\mathrm{a}}$ & 0,93 & 0,93 & 28 & 20 & 0,94 & 0,93 & 34 & 28 & 0,92 & 0,92 & 26 & 19 \\
\hline & $4^{\mathrm{a}}$ & 0,88 & 0,84 & 52 & 44 & 0,90 & 0,88 & 44 & 36 & 0,91 & 0,92 & 34 & 22 \\
\hline & $5^{\mathrm{a}}$ & 0,90 & 0,86 & 65 & 55 & 0,91 & 0,98 & 55 & 43 & 0,92 & 0,92 & 47 & 36 \\
\hline \multirow{5}{*}{ PT } & $1^{\mathrm{a}}$ & 0,80 & 0,89 & 6 & 0 & 0,78 & 0,87 & 6 & -1 & 0,68 & 0,80 & 7 & -3 \\
\hline & $2^{\underline{a}}$ & 0,84 & 0,91 & 8 & -2 & 0,87 & 0,92 & 6 & 1 & 0,86 & 0,91 & 8 & -3 \\
\hline & $3^{\mathrm{a}}$ & 0,91 & 0,91 & 30 & 17 & 0,92 & 0,91 & 40 & 32 & 0,89 & 0,90 & 29 & 19 \\
\hline & $4^{\mathrm{a}}$ & 0,86 & 0,84 & 59 & 42 & 0,85 & 0,83 & 56 & 40 & 0,87 & 0,90 & 42 & 22 \\
\hline & $5^{\mathrm{a}}$ & 0,87 & 0,83 & 78 & 59 & 0,87 & 0,96 & 69 & 49 & 0,89 & 0,89 & 56 & 38 \\
\hline \multirow{5}{*}{$\mathrm{RS}$} & $1^{\mathrm{a}}$ & 0,57 & 0,75 & 9 & 2 & 0,54 & 0,67 & 9 & -4 & 0,42 & 0,65 & 9 & -1 \\
\hline & $2^{\underline{a}}$ & 0,70 & 0,83 & 11 & 1 & 0,74 & 0,84 & 7 & -1 & 0,72 & 0,84 & 9 & -1 \\
\hline & $3^{\mathrm{a}}$ & 0,75 & 0,85 & 33 & 4 & 0,80 & 0,94 & 25 & 7 & 0,73 & 0,84 & 31 & 4 \\
\hline & $4^{\mathrm{a}}$ & 0,63 & 0,70 & 76 & 47 & 0,72 & 0,82 & 54 & 29 & 0,67 & 0,79 & 56 & 23 \\
\hline & $5^{\mathrm{a}}$ & 0,71 & 0,82 & 66 & 24 & 0,75 & 0,97 & 55 & 18 & 0,73 & 0,83 & 58 & 16 \\
\hline \multirow{5}{*}{ RSS } & $1^{\mathrm{a}}$ & 0,74 & 0,71 & 13 & 10 & 0,66 & 0,80 & 7 & 1 & 0,66 & 0,71 & 11 & 7 \\
\hline & $2^{\mathrm{a}}$ & 0,76 & 0,57 & 26 & 24 & 0,78 & 0,68 & 15 & 13 & 0,50 & 0,51 & 27 & 20 \\
\hline & $3^{\mathrm{a}}$ & 0,84 & 0,82 & 45 & 34 & 0,88 & 0,91 & 39 & 31 & 0,82 & 0,80 & 42 & 32 \\
\hline & $4^{\mathrm{a}}$ & 0,71 & 0,60 & 102 & 88 & 0,72 & 0,65 & 78 & 61 & 0,76 & 0,71 & 74 & 60 \\
\hline & $5^{\mathrm{a}}$ & 0,79 & 0,67 & 112 & 100 & 0,84 & 0,94 & 91 & 80 & 0,81 & 0,73 & 93 & 79 \\
\hline
\end{tabular}

De forma geral, verifica-se que os coeficientes de precisão (r) e de exatidão (d) apresentaram valores em torno de 0,9 para os métodos PMAP e PMAPS, e que seus valores diminuíram à medida que as estimativas foram realizadas pelos métodos PMF ou pelos que utilizam somente dados de radiação solar e/ou temperatura do ar (PT, RS e RSS). Com relação aos coeficientes RMSE e MBE, observa-se que o erro de precisão de todos os métodos avaliados foi, no máximo, $10 \mathrm{~W} \mathrm{~m}^{-2}$ nas duas 
primeiras etapas de medição. Com o aumento da $\mathrm{ET}_{\mathrm{c}}$, resultante da expansão foliar, o erro de precisão aumentou nas etapas seguintes, ficando, respectivamente, entre 20 e $54 \mathrm{~W} \mathrm{~m}^{-2}$ para os métodos PMF, PMAP e PMAPS e entre 24 e $75 \mathrm{~W} \mathrm{~m}^{-2}$ para PT, RS e RSS. Com relação à exatidão, nota-se que, independentemente da cultivar, o MBE foi menor que $2 \mathrm{~W} \mathrm{~m}^{-2}$ nas duas primeiras etapas de medição quando foram utilizados os métodos PMAP e PMAPS. Para as etapas seguintes, o MBE foi positivo, com base nas estimativas obtidas com PMAP, PMAPS, PT e RS, indicando que esses métodos superestimaram a $\mathrm{ET}_{\mathrm{c}}$. Em contraste, o método PMF subestimou a ETc para a cultivar Great Lakes em todas as etapas de medição.

Nas Figuras 1 e 2, apresenta-se a variação diurna dos valores medidos e estimados de $\mathrm{ET}_{\mathrm{c}}$ para o $5^{\mathrm{o}}$ e $25^{\circ}$ dias após o transplantio, quando os IAFs médios para as três cultivares eram $0,12 \mathrm{e}$ 3,14 , respectivamente. Para facilitar a análise de desempenho dos diferentos métodos de estimativa de $\mathrm{ET}_{\mathrm{c}}$, a marcha diurna do $\mathrm{DPV}_{\mathrm{ar}}$ também foi plotada em cada um dos gráficos mostrados nessas figuras.

No período inicial de crescimento (5 DAT), independentemente da cultivar, observa-se que o método PMF estimou a $\mathrm{ET}_{\mathrm{c}}$ de forma satisfatória no período da manhã, mas não foi capaz de incorporar os efeitos do aumento do $\mathrm{DPV}_{\text {ar }}$ na evapotranspiração à tarde, a qual foi severamente subestimada. Entretanto, com o aumento expressivo do IAF, observado no 25 DAT, o erro de estimativa da $\mathrm{ET}_{\mathrm{c}}$ com o método PMF foi atenuado pela diminuição do $\mathrm{DPV}_{\mathrm{ar}}$, resultando da maior liberação de vapor de água no ambiente de cultivo. Esse desempenho deficiente do método PMF em ambiente protegido, especialmente no período inicial de crescimento, pode ser atribuído à parametrização do modelo de PenmanMonteith, conforme sugerido por ALLEN et al. (1998).

Como a velocidade do ar é extremamente baixa em condições de ambiente protegido, a relação 208/ $U_{2}$ superestima excessivamente o parâmetro $r_{a}$, que, por sua vez, subestima a importância do termo aerodinâmico desse modelo.

Por outro lado, independentemente da cultivar e do estágio de crescimento da cultura, o método PMAP, bem como a sua versão simplificada (PMAPS), possibilitaram estimativas mais próximas dos valores observados de $\mathrm{ET}_{\mathrm{c}}$, tanto no período da manhã como no da tarde. Esses resultados sugerem que, quando o modelo de Penman-Monteith é adequadamente parametrizado, os dados dos sensores de umidade relativa e de velocidade do ar não são cruciais para boas estimativas horárias da $\mathrm{ET}_{\mathrm{c}}$ da alface cultivada em sistema hidropônico sob ambiente protegido. As condições micrometeorológicas específicas dos ambientes protegidos, como velocidade do ar abaixo de $0,5 \mathrm{~m} \mathrm{~s}^{-1}$ (HANAN, 1998) e umidade relativa do ar próxima à saturação no início do período da manhã (BURIOL et al., 2000) permitem versões simplificadas do método de Penman-Monteith, contribuindo, dessa forma, para a redução dos custos de implantação de sistemas automáticos para aplicação da solução nutritiva. No entanto, deve-se observar que ambos os métodos PMAP e PMAPS ainda requerem medições ou estimativas do saldo de radiação à superfície do dossel vegetativo.

Similarmente ao PMF, os métodos baseados na equação de Priestley-Taylor (PT), na radiação solar global e temperatura do ar (RS) e na temperatura do ar exclusivamente (RSS) não tiveram bom desempenho na estimativa da $\mathrm{ET}_{\mathrm{c}}$ após o meio-dia, durante o período inicial de crescimento da alface. Entretanto, com a diminuição do $\mathrm{DPV}_{\text {ar }}$ no final do ciclo da cultura, chegando no máximo a $1.400 \mathrm{~Pa}$, aproximadamente, o desempenho dos métodos PT e RS melhorou no período da tarde. Porém, esse fato não foi observado com o método RSS, que se baseia somente na temperatura e, assim, a $\mathrm{ET}_{\mathrm{c}}$ continuou a ser subestimada nesse período. Finalmente, outro fato a ser destacado, que pode ser observado tanto no início como no final do ciclo de crescimento da alface, é que os métodos baseados na radiação solar e temperatura do ar (PT, RS e RSS) tenderam a superestimar a $\mathrm{ET}_{\mathrm{c}}$ no início do período da manhã. 

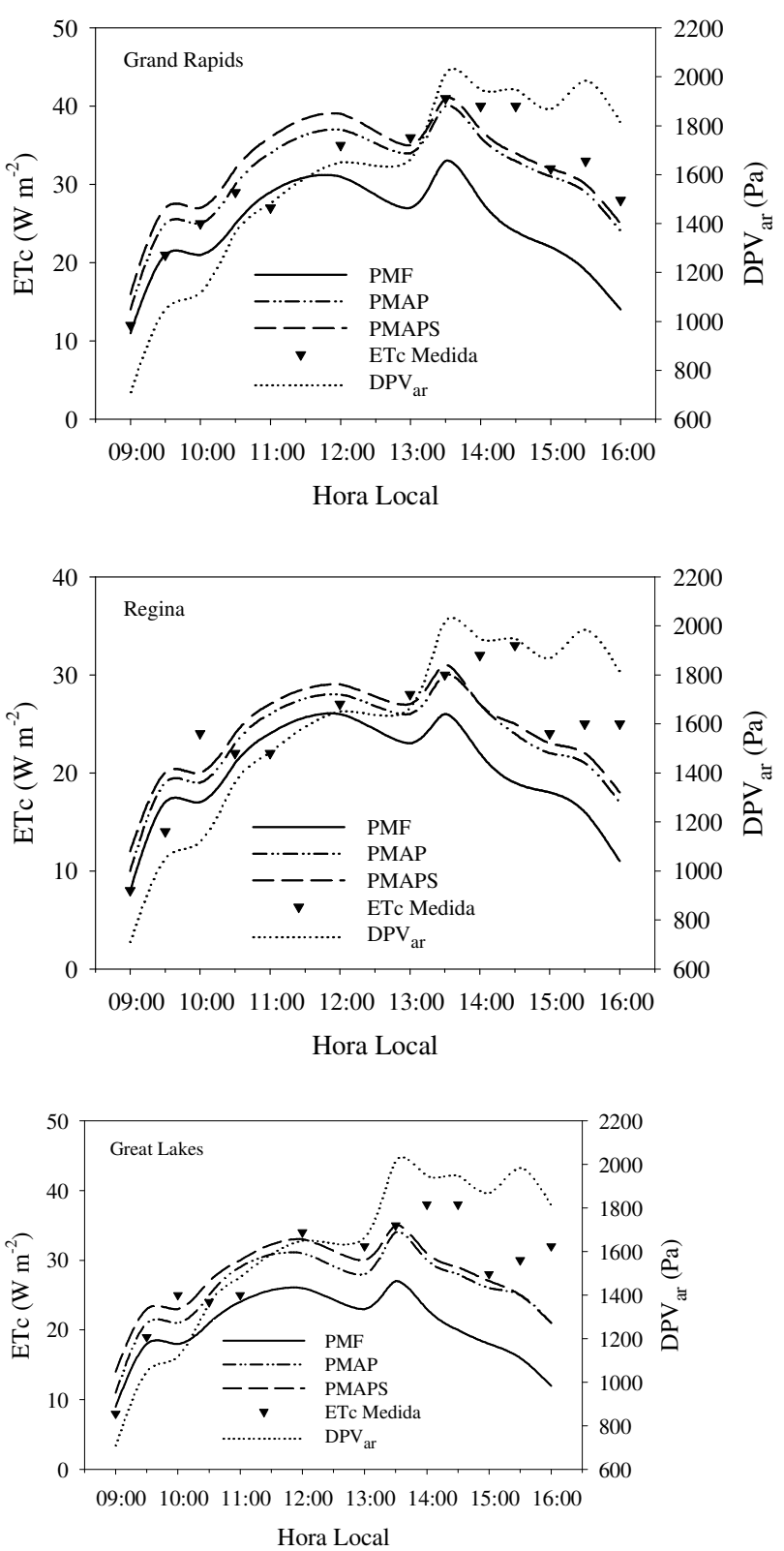
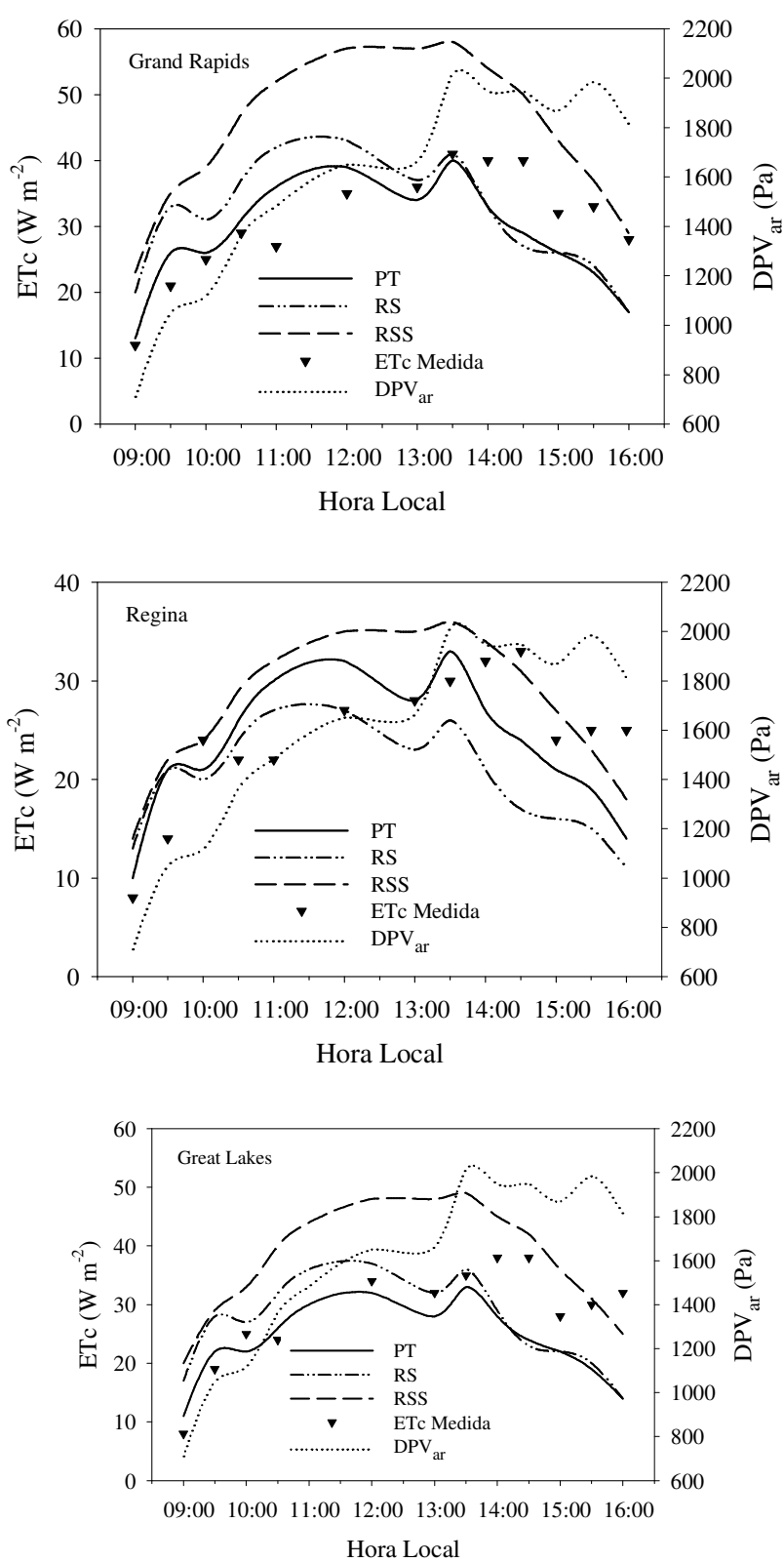

FIGURA 1. Variação diurna dos valores medidos e estimados da evapotranspiração $\left(\mathrm{ET}_{\mathrm{c}}\right)$ de três cultivares de alface hidropônica sob ambiente protegido (eixo esquerdo) e alterações correspondentes do déficit de pressão de saturação de vapor de água no ar (eixo direito). As medições e estimativas de $\mathrm{ET}_{\mathrm{c}}$ foram realizadas no $5^{\underline{\mathrm{o}}}$ dia após o transplantio, por meio dos métodos Penman-Monteith-FAO (PMF), Penman-Monteith Modificado para Ambientes Protegidos (PMAP) e PMAP Simplificado (PMAPS), apresentados no lado esquerdo, e Priestley-Taylor (PT), Radiação Solar (RS) e RS Simplificado (RSS), ilustrados no lado direito. Diurnal variation of the measured and estimated values of evapotranspiration $\left(E T_{c}\right)$ for three hydroponic lettuce cultivars under greenhouse conditions (left axis) and corresponding alterations of the air vapor pressure deficit (right axis). Measurements and estimates of ET $_{c}$ were carried out on the $5^{\text {th }}$ day after transplanting, by means of the methods Penman-Monteith-FAO (PMF), Penman-Monteith Modified for greenhouse conditions (PMAP) and Simplified PMAP (PMAPS), presented on the left side, and Priestley-Taylor (PT), Solar Radiation (RS) and Simplified RS (RSS), illustrated on the right side. 


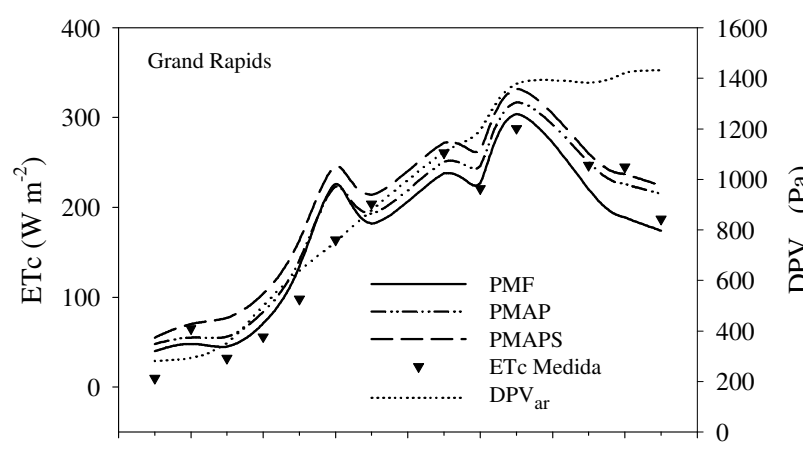

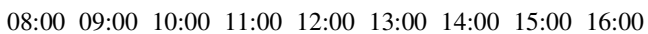

Hora Local

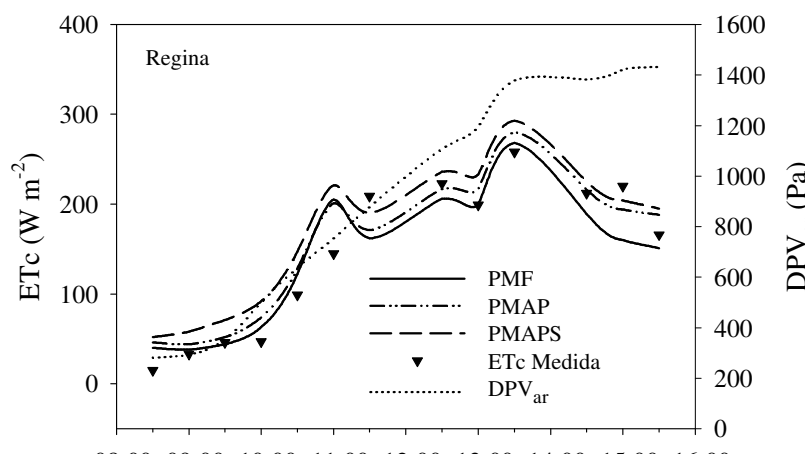

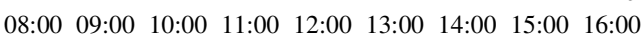

Hora Local

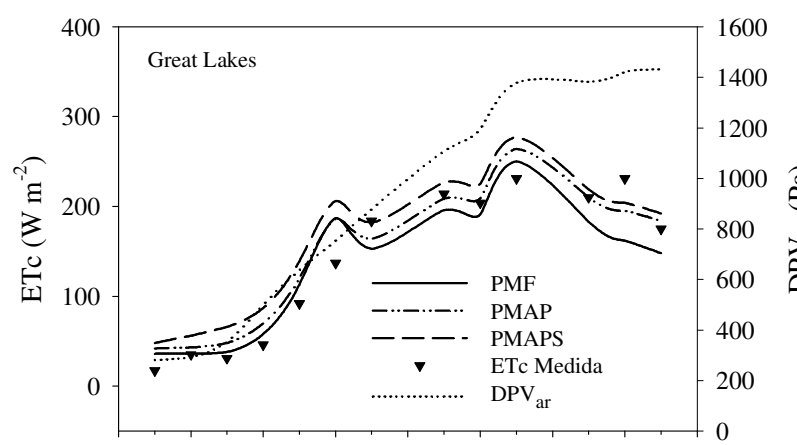

08:00 09:00 10:00 11:00 12:00 13:00 14:00 15:00 $16: 00$

Hora Local

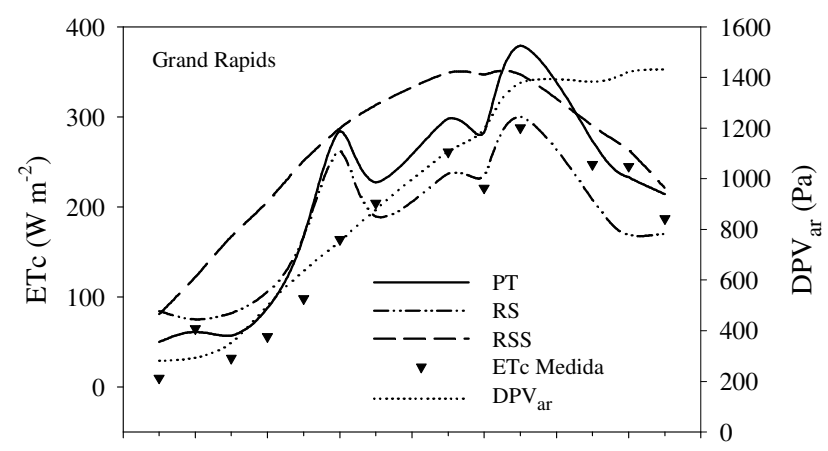

$\begin{array}{llllllllllllllll}08: 00 & 09: 00 & 10: 00 & 11: 00 & 12: 00 & 13: 00 & 14: 00 & 15: 00 & 16: 00\end{array}$

Hora Local

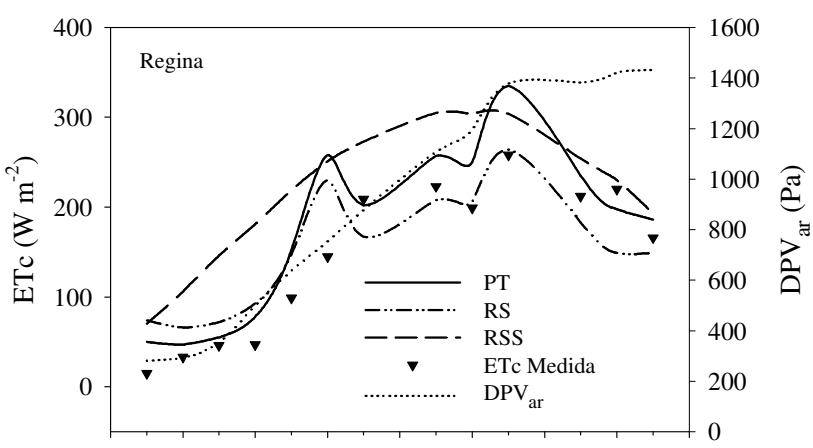

$\begin{array}{lllllllllll}08: 00 & 09: 00 & 10: 00 & 11: 00 & 12: 00 & 13: 00 & 14: 00 & 15: 00 & 16: 00\end{array}$

Hora Local

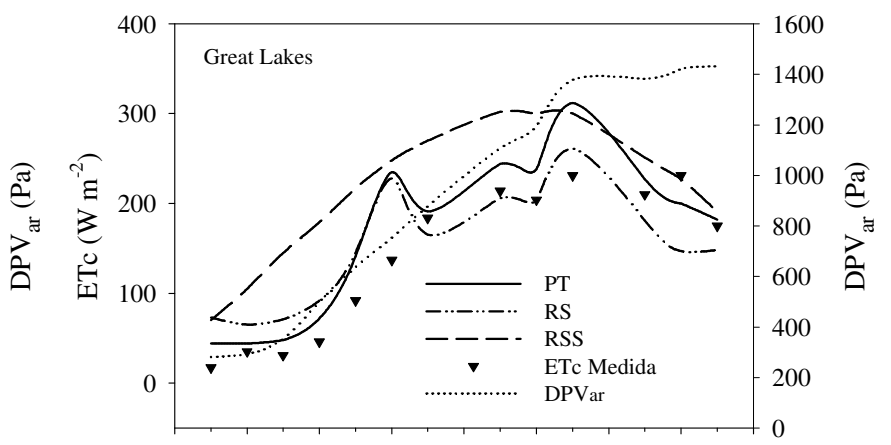

08:00 09:00 10:00 11:00 12:00 13:00 14:00 15:00 $\quad 16: 00$

Hora Local

FIGURA 2. Variação diurna dos valores medidos e estimados da evapotranspiração $\left(\mathrm{ET}_{\mathrm{c}}\right)$ de três cultivares de alface hidropônica sob ambiente protegido (eixo esquerdo) e alterações correspondentes do déficit de pressão de saturação de vapor de água no ar (eixo direito). As medições e estimativas de $\mathrm{ET}_{\mathrm{c}}$ foram realizadas no $25^{\mathrm{o}}$ dia após o transplantio, por meio dos métodos Penman-Monteith-FAO (PMF), Penman-Monteith Modificado para Ambientes Protegidos (PMAP) e PMAP Simplificado (PMAPS), apresentados no lado esquerdo, e Priestley-Taylor (PT), Radiação Solar (RS) e RS Simplificado (RSS), ilustrados no lado direito. Diurnal variation of the measured and estimated values of evapotranspiration $\left(\mathrm{ET}_{\mathrm{c}}\right)$ for three hydroponic lettuce cultivars under greenhouse conditions (left axis) and corresponding alterations of the air vapor pressure deficit saturation (right axis). Measurements and estimates of $\mathbf{E T}_{\mathrm{c}}$ were carried out on the $25^{\text {th }}$ day after transplanting, by means of the methods Penman-Monteith-FAO (PMF), Penman-Monteith Modified for greenhouse conditions (PMAP) and Simplified PMAP (PMAPS), presented on the left side, and Priestley-Taylor (PT), Solar Radiation (RS) and Simplified RS (RSS), illustrated on the right side. 


\section{CONCLUSÕES}

O método de Penman- Monteith, parametrizado conforme sugerido por ALLEN et al. (1998), é inapropriado para estimativa de valores horários de evapotranspiração da alface hidropônica em ambiente protegido.

A parametrização aerodinâmica proposta por McNAUGHTON \& JARVIS (1983), baseada no fator de desacoplamento, possibilita melhores estimativas da $\mathrm{ET}_{\mathrm{c}} \mathrm{e}$, adicionalmente, dispensa medições da velocidade do ar em ambiente protegido (método PMAP). Adicionalmente, desde que o $\mathrm{DPV}_{\mathrm{ar}}$ seja estimado corretamente, medições da umidade relativa do ar não são estritamente necessárias para estimativas satisfatórias da $\mathrm{ET}_{\mathrm{c}}$ em ambientes protegidos (método PMAPS).

A utilização de métodos baseados na radiação solar incidente e/ou temperatura do ar não se mostrou adequada para estimativa da $\mathrm{ET}_{\mathrm{c}}$, principalmente no período da tarde. Esse fato foi agravado no período inicial de crescimento da cultura. Em contraste, os métodos PMAP e PMAPS possibilitaram estimativas mais adequadas da $\mathrm{ET}_{\mathfrak{c}}$, tanto no período da manhã como no da tarde.

\section{REFERÊNCIAS}

ALLEN, R.G.; PEREIRA, L.S.; RAES, D., SMITH, M. Crop evapotranspiration: guidelines for computing crop requirements. FAO: Rome, 1998. 300 p. (Irrigation and Drainage Paper, 56).

BURIOL, G.A.; RIGHI, E.Z.; SCHNEIDER, F.M.; STRECK, N.A.; HELDWEIN, A.B.; ESTEFANEL, V. Modificação da umidade relativa do ar pelo uso e manejo da estufa plástica. Revista Brasileira de Agrometeorologia, Santa Maria, v.8, n.1, p.11-18, 2000.

FARIAS, J.R.B.; BERGAMAMASCHI, H.; MARTINS, S.R.; BERLATO, M.A.; OLIVEIRA, A. C. B. Alterações na temperatura e umidade relativa do ar provocadas pelo uso de estufa plástica. Revista Brasileira de Agrometeorologia, Santa Maria, v.1, n.1, p.51-62, 1992.

FELTRIN, A.L.; CECÍLIO FILHO, A.B.; BRANCO, R.B.F.; BARBOSA, J.C.; SALATIEL, L.T. Produção de alface-americana em solo e em hidroponia, no inverno e verão, em Jaboticabal - SP. Revista Brasileira de Engenharia Agrícola e Ambiental, Campina Grande, v.9, n.4, p.505-509, 2005.

GUALBERTO, R.; OLIVEIRA, P.S.R.; GUIMARÃES, A.M. Adaptabilidade e estabilidade fenotípica de cultivares de alface do grupo crespa em cultivo hidropônico. Horticultura Brasileira, Brasília, v.27, n.1, p.7-11, 2009.

HANAN, J.J. Greenhouses - Advanced technology for protected horticulture. Boca Raton: CRC Press LLC, 1998. 684 p.

HARGREAVES, G.H.; SAMANI, Z.A. Estimating potential evapotranspiration. Journal of Irrigation and Drainage Engineering, New York, v.108, p.225-230, 1982.

JACOVIDES, C.P.; KONTOYIANNIS, H. Statistical procedures for the evaluation of evapotranspiration computing models. Agricultural Water Management, Amsterdam, v.27, p.365$371,1995$.

JONES, J.B., Jr. Hydroponics: A practical guide for the soilless grower. Boca Raton: St. Lucie Press, 1997. $230 \mathrm{p}$.

LUZ, J.M.Q.; GUIMARÃES, S.T.M.R.; KORNDÖRFER, G.H. Produção hidropônica de alface em solução nutritiva com e sem silício. Horticultura Brasileira, Brasília, v.24, n.3, p.295-300, 2006.

LYRA, G.B.; ZONIER, S.; COSTA, L.C.; SEDIYAMA, G.C.; SEDIYAMA, M.A.N. Modelos de crescimento para alface (Lactuca sativa L.) cultivada em sistema hidropônico sob condições de casa de vegetação. Revista Brasileira de Agrometeorologia, Santa Maria, v.11, n.1, p.69-77, 2003.

LYRA, G.B. Estimativa da evapotranspiração e análise de crescimento para alface (Lactuca sativa L.) cultivada em sistema hidropônico em condições de casa de vegetação. 2002 . $91 \mathrm{f}$. Dissertação (Mestrado em Meteorologia Agrícola) - Universidade Federal de Viçosa, Viçosa - MG, 2002. 
MAGGI, M.F.; KLAR, A.E.; JADOSKI, C.J.; ANDRADE, A.R.S. Produção de variedades de alface sob diferentes potenciais de água no solo em ambiente protegido. Irriga, Botucatu, v.11, n.3, p.415427, 2006.

McNAUGHTON, K.G.; JARVIS, P.G. Predicting effects of vegetation changes on transpiration and evaporation. In: KOZLOWSKI, T.T. (Ed.). Water deficits and plant growth. New York: Academic Press, 1983. v.2, p.1-47.

MONTEITH, J.L. Evaporation and environment. Symposium of the Society for Experimental Biology, London, v.19, p.205-234, 1965.

PEREIRA, A.R.; VILLA NOVA, N.A.; SEDIYAMA, G.C. Evapo(transpi)ração. Piracicaba: FEALQ, 1997. 183 p.

SILVA, J.O.; SOUZA, P.A.; JÚNIOR, J.G.; PEREIRA, P.R.G.; ROCHA, F.A. Crescimento e composição mineral da alface no sistema hidropônico por capilaridade. Irriga, Botucatu, v.10, n.2, p.146-154, 2005.

WILLMOTT, C.J. On the validation of models. Physical Geography, Columbia, v.2, p.184-194, 1981.

ZOLNIER, S.; LYRA, G.B.; GATES, R.S. Evapotranspiration estimates for greenhouse lettuce using an intermittent nutrient film technique. Transactions of the ASAE, St. Joseph, v.47, n.1, p.271-282, 2004. 